\title{
Of Apps and the Menstrual Cycle: A Journey into Self-Tracking
}

Jacqueline Gaybor

\section{Tracking the Origins of This Chapter}

The use of mobile apps for self-monitoring of health and well-being has grown exponentially in the last years, reaching 6.1 billion users by 2020 (Statista, 2020). A major attraction of self-monitoring health-related aspects such as food consumption, hours of sleep, weight changes, physical activity, or the menstrual cycle through digital technologies is to use personal information to achieve health-awareness and self-improvement, defined as "the belief that the self-knowledge that will eventuate will allow self-trackers to exert greater control over their destinies" (Lupton, 2016: 115). Digitized strategies for self-tracking the menstrual cycle, the focus of this chapter, is a subgroup of health apps developed to track a person's menstrual cycle, sex life, and health to provide them with algorithmically derived insights into their body and lives.

J. Gaybor $(\bowtie)$

Rutgers Kenniscentrum Seksualiteit, Utrecht, The Netherlands e-mail: j.gaybor@rutgers.nl 
I have wanted to investigate menstrual-tracking apps since I was finishing with my doctoral thesis on the body politics of menstruation and reusable technologies for menstrual management in 2019. At first, I conceived this research from a different angle than the one I have eventually used in this chapter. My interest initially was to explore whether menstrual-tracking apps (MTA) developers were fulfilling their legal obligations under the General Data Protection Regulation (GDPR). I wanted to research how menstrual-tracking apps were processing user data and what data was collected and stored. From a feminist political perspective, I felt it was important to research issues of security and privacy related to the menstruating body in online spaces. I had serious concerns, especially around consent, privacy and transparency in relation to how personal data, specifically medical data, is handled by these companies. Just to give an idea, Privacy International (PI), an organization based in the UK focussed on the promotion of the right to privacy, found that of the 36 apps tested in their study, 61\% automatically transferred data to Facebook the moment a user opened the app. This happened regardless of "whether the user had a Facebook account or not, and whether they [were] logged into Facebook or not" (Privacy International, 2020).

At the same time, on a personal level, and because of my Ph.D. research on the body politics of menstruation, I found myself more and more frequently involved in conversations about the use of these apps with my friends, family and colleagues. While there was somehow a recognition of the risks to privacy by filling personal data into the app, other dimensions were often highlighted. They emphasized the positive gain in knowledge about their body and learning about their menstrual cycle. In these conversations it was automatically assumed that I was a user of a MTA, and on numerous occasions I was asked which MTA is the best, my (presumed) experience in tracking my menstrual cycle through an app, or whether I ever felt it had helped me justify my behaviour or enhanced my body awareness. Or if I ever used the app to validate certain feelings and emotions at a specific time of the month.

My experience? Well, I began to wonder, how would I know? Should I know? Before conducting this investigation, I had never used any form of health tracking app, including the MTA. My distrust towards how medical data is handled by app companies, which motivated my interest in researching MTAs, was an important reason that kept me away from using them. I did not find it appealing to use digital technologies to self-monitor my body and my menstrual cycle, probably because I have 
always had very regular periods. I used the old fashioned "analogue" menstrual-tracking method through which I rely on my memory or pen and agenda.

Nevertheless, I became puzzled by the questions I was asked and started to reflect on the possibilities of building body awareness through a digital technology. I was interested to know how the different interactions between the user and the MTA could give rise to different forms of experiencing the body and the menstrual cycle. Could selfknowledge be gained by sharing, then collecting, and ultimately reflecting on information produced by the MTA out of the user's data? Could my relationship with my body be reconfigured through a self-tracking app that is powered by my personal digital traces? And most importantly, how should I approach this exploration? While such questions motivated me and guided me throughout this journey, they will be addressed in a somewhat different way. Rather than theorizing and problematizing the results of my journey while using the app, I focus on the process of knowledge production grounded in my own messy experience of familiarizing myself with the world of MTAs by becoming a user of one of them.

In this chapter, I describe and reflect on this journey, which I took from the summer of 2019 to the winter of 2021. The first stage, which I call "the overwhelming period" is when I first approached these apps, and where my personal bias about the MTA combined with my training as a lawyer complicated the process of adopting the use of an MTA. Then, I moved into a different stage, characterized by intimate encounters with others, which I call "analogue intimacy". In this stage, I left my individual research quest and interaction with the digital realm and found myself sharing emotional and embodied life stories with friends who are users of MTAs. These encounters pushed me to question my own preconceptions and biases. In the next stage, called "Flo and me", I share my first impressions of Flo, the MTA I ended up adopting for this journey. During this last stage of the journey, "the production of disembodied knowledge of the body", I reflect on the tensions and dilemmas I encountered as a user of Flo. In "final thoughts", I highlight the importance of creating "dedicated times, spaces and contexts within which to be reflexive" (Mauthner \& Doucet, 2003: 425) in an effort to be transparent about how my pre-conceived ideas and assumptions influenced the process of knowledge construction and production.

To document the MTA journey, I used a "field" diary, the field being mostly located in my house in The Hague, where I have spent most of 
my time during the Covid-19 quarantine in 2020 and 2021. In this diary I documented my embodied and emotional experiences, which I consider central to the process of knowledge production. This has been a crucial aid for my memory and has supported my process of reflexivity as a tool "for analysis whereby the representation of this process of understanding is as important as the product of this process of knowledge production" (Willemse, 2014: 38).

\section{Positioning THE JOURneY}

In qualitative social science research, there is a general acknowledgement that the interpretation of data is a reflexive exercise through which meanings are creatively constructed rather than found (Mauthner \& Doucet, 2003). Within discussions of reflexivity, feminist scholars (Grosz, 1994; Harding, 2004) have drawn attention to the importance of recognizing the researchers' epistemological and ontological positions and their social and emotional locations and how they influence the process of knowledge production. Feminist methodological critiques have thus rejected the positivist notion of the neutral status of the researcher and recognized the partial and provisional nature of knowledge claims (Letherby, 2002; Mauthner \& Doucet, 2003). In this chapter, I engage in a feminist methodological exploration by detaching from notions of the neutral and objective researcher. I draw on my personal and messy story, making explicit my social position, interests, and background assumptions, and writing about these in the first person. The choice of revealing rather than covering up the messiness of my lived experiences in my writing is as Ellingson (1998) describes, a way to debunk positivist assumptions about a researcher's neutrality and objectivity with the clear intention of "positioning scholars as imperfect social actors" (Ellingson, 2006: 299). This required me to go beyond just stating my own biases and pushed me to look carefully at how different influences, life experiences, including multiple discussions, emotional conversations, and how the writing and later reading and reflecting on my field diary affected this process.

My methodological choices explain why I found myself frequently involved in conversations about the use of MTAs. In addition to defining 
myself as a cis-woman ${ }^{1}$ and of an age where people menstruate, for more than a decade I have been involved with menstrual activism in Ecuador, where I am from, and in Argentina, where I conducted my Ph.D. research between 2015 and 2020. As part of my involvement with menstrual activism, I have frequently attended get-togethers on DIY reusable technologies, self-management gynaecology courses, menarche welcome ceremonies, and painting workshops with menstrual blood, among others. All these experiences have shaped my relationship with my body and my menstrual cycle, as well as inspired my Ph.D. research. During these years, I have investigated various reusable technologies for managing menstruation, not only from the comfort of my desk in The Netherlands, where I have been based since 2012, but through my own embodied experience, as a user of these technologies. I agree with Ellingson (2017) that a researcher's body plays an important role in producing all types of qualitative research. It is through the body, understood as "simultaneously physical and affective, social and individual, produced and producing, reproductive and innovative" (Jones \& Woglom, 2015: 116) that knowledge is produced. The body as stated by Ellingson (2017) is inseparable from the self that produces knowledge.

In a previous work (Gaybor, 2019), I (self-)explored the menstrual body (using my own) as a site of knowledge production. My body was engaged in all aspects of the research process. By using certain reusable menstrual technologies (such as the menstrual cup, reusable panties, and pads), my body became a known and inhabited space, with temperature, texture, and a 3D depth. This (self-)exploration has been key in my understanding and development of body awareness through the assemblage of my body with technological artefacts. In the MTA journey, I also engaged, although differently, with digital technologies: social media platforms, like blogs, Instagram Live sessions, and Facebook closed groups. This engagement pushed me to recognize the potential of digital media culture in pushing forward the political agenda on the body politics of menstruation (Gaybor, 2020). However, as I mentioned above, my concerns about privacy, surveillance, and forms of monetizing information have unquestionably lowered my optimistic vision about social media and its role in addressing key feminist political issues. Facebook, for

${ }^{1}$ I recognize that not all people who menstruate are women and that not all women menstruate. As a biological event, menstruation can be experienced by women, girls, and other bodies and identities. I find the term "menstruators" (Bobel, 2007) useful here. 
instance, has been criticized for invading user privacy through its continuous surveillance for commercial and political purposes (Shaw, 2016), or Instagram, for the censorship of certain information over other information (Kaur, 2015). Furthermore, I was not eager to include MTAs in my previous self-exploratory journey with menstrual technologies since I was concerned with privacy violations when personally identifiable data is collected through digital technologies (Mahdawi, 2019; Privacy International, 2018; Shipp \& Blasco, 2020).

\section{The Overwhelming Period}

Since I embarked on this research, I knew that defining which MTA to use was going to be challenging. But I did not envision how challenging this was going to be. To begin, there were many options, which I found overwhelming. Moreover, while there was a great variety regarding the number of apps, the same cannot be said regarding the type of subject that is continuously promoted in these apps. My observations fit what scholars (Lupton, 2015; Thomas \& Lupton, 2015) have argued: that MTAs are mostly advertised to a white, heterosexual cis-woman. This became evident when looking at the type of interface (photographs or designs) that these apps use. They portray beautiful mostly white women, in their $20 \mathrm{~s}$ and 30 s and mostly thin. There was an emphasis on talking about conception and romantic or sexual relationships, but from a heterosexual perspective. The next hurdle I encountered concerned the commercialization of these apps. While there were MTAs that were free to download, most of them had paid upgraded versions, which supposedly gave access to top services. After these two first filters, I spent a couple of weeks reading some of the apps' privacy policies. This stage took much longer than planned. My previous training as a lawyer emerged and with it my scrupulous attention to detail. In general, I was alarmed to see how short or general these policies were, providing almost no information about how data was handled and processed by the developer. In some cases, what struck me was the lack of clarity and the use of ambiguous language. Most of these policies were, to my view, strategically written to create uncertainty. Keywords such as "possibly", "may", or "potentially" were frequently used and linked to the actions of the developers. I commonly found sentences structured as follows: "When you access or use the App, we may automatically collect the following information: $[\ldots]$ ", or "When 
you sign up to use the App, we may collect Personal Data about you such as $[\ldots] "$.

This both disappointed and worried me. All the privacy policies I reviewed were difficult to understand. They used verbose, unintelligible and confusing language. As I feared, I did not find one MTA with which I could feel comfortable or at least safe to start this exploration. As I navigated these confusing waters, trying to seek clarity and devoting time to learn about GDPR and privacy protection, without noticing, I was deviating from what I wanted to write in this piece. At this stage, I wondered: what is the purpose of this investigation? Should I be rather writing about data privacy or embarking on a legal exploration on how MTA developers are obtaining, using and handling user's data? At the same time, I was puzzled. There is an abundance of MTAs. Millions of people have installed them on their phones. They voluntary share their private and intimate everyday life information: the day they menstruate, the consistency and quantity of the discharge, when they have sex, whether they use protection, or their mood swings, etc. It is difficult for me to picture people sharing this information in an analogue way with complete strangers. The stigma and taboo that surrounds this topic normally prevents having these conversations even with close friends or family members. At what point does sharing this information lose its intimate aspect? Is it because an app is distanced from the physical body? Does this act of separating private information from the physical body, the analogue body, allow it to feel anonymized, despite the reality that the information can potentially be accessed by others? This made me think that MTAs might be offering users an opportunity to escape, yet not challenge the stigma associated with menstruation. On the other hand, Karlsson (2019) argues that privacy concerns do not seem to play a significant role in influencing the use of MTAs. She states that when the female body is put into data through MTAs, users expect to disappear in the abundance of data, assuming that the information that corresponds to their bodies cannot be traced to them. This emphasizes why "privacy continues to be such an important issue for feminist theory, beginning with the attack on the public/private divide and progressing to an ongoing investigation of privacy related to the female body in offand online spaces" (Karlsson 2019: 120). 


\section{Analogue Intimacy}

After an intense initial phase of reading the apps' privacy policies, familiarizing myself with what had been published in the context of mobile health and MTAs, and imagining my personal experience of using them, I started to speak about this topic with friends and family who were long-term users of these apps. Reading my diary back, I see that this process was challenging as I struggled to cope with my own prejudices of the apps, the emotions of helplessness that I felt when reading the privacy policies and at the same time listening to different and more positive perspectives and views regarding MTAs. I see how listening to people who have lived through the experience of using the apps, was an important step in my overall reflective process. As Sörensson and Kalman (2017) state, knowledge is developed in connection and dialogue with others of a community, not in isolation from them.

As I took part in these dialogues with users of MTAs, I often found a common language and relatable menstrual stories. This, I believe, is partly because users of MTAs belong to a specific minority, mostly young, socioeconomically privileged, health-conscious and technologically oriented (Lupton, 2016). It is a privileged minority of which I am part. I could relate to some of these stories, I noted peoples' tears and feelings of despair that I could understand and empathize, even if not personally relate to from my own experience of menstruation. Intense physical pain because of endometriosis, or emotional wounds because of health problems that led to problems of conceiving, were among the reasons that people had begun to use the apps. These were not my reasons.

Here it is probably important to say that MTAs are promoted as technologies that allow for a close observation and monitoring of the cycle, measurement of other vital signs, and support with the detection and estimation of the ovulation timing, which is critical for conception. Observing and having access to processed personal data, I heard on multiple occasions, helped to see patterns, understand symptoms, and sometimes explain causes that could lead to a diagnosis. MTAs were used as normative standards of comparison.

When I worked with reusable menstrual technologies, I found engaging in dialogue with others about MTAs was incredibly enriching. It brought me closer to people; to an intimate space where we could both share and listen. These narratives, where people shared their lives, both in the past and in the present made me realize how fortunate I was to 
enjoy a healthy body. It was perhaps for this reason that I did not see the need to use MTAs earlier. These conversations were an important step into questioning my vision of these apps as superfluous, which I became conscious of while in dialogue with others. I had to learn to be aware of my own biases about the apps and leave my prejudice aside in order to understand other people's perspectives and embodied experiences when using them.

Revisiting my diary notes, I see how my perspective on these apps has been continually nuanced as more questions emerge with the passing of the days. This diary turned out to be an important document for tracking, in an analogue way, my thought process and emotions throughout this research journey. I began to better understand how the automated interpretation of personal data could be informative and even necessary for some health purposes. In cases where conception was desired, the MTAs represented something more than a digital tracking method, they were a form of materialization of hope. Something to hold on to in extremely difficult and emotionally draining circumstances.

I was navigating the tension between the optimistic outlook, which endorses the resounding celebration of MTAs from medical professionals, fashion, and beauty magazines. MTAs have been widely advertised as being able to revolutionize health care, medical research and help to achieve well-being (Sanders, 2017). This narrative reinforces the idea that having the possibility of knowing more about ourselves and our bodies through data can be experienced as powerful, educational and can lead towards self-improvement (Lupton, 2016). This positive outlook was contrasted in my mind with a more careful and critical stand that questions the insidious effects of MTAs in placing an unnecessary burden on people's lives and on commodifying their personal data. Along similar lines, I found myself questioning, more and more, the presumed benefits of neutrality offered by digital data and their accuracy, for instance, in diagnosing and in predicting fertile windows to aid conception (Setton et al., 2016). How to balance the digital data vis-a-vis people's own embodied knowledge of their bodies? 


\section{FLO AND ME}

Despite the hesitations outlined above, I became one of the "over 130 million women" 2 users of Flo around the globe (Flo, 2020). For the readers who are not familiar with Flo, I would like to introduce the app briefly as part of the contextualization of my research journey. The app offers two versions: free and unlimited access. The free version offers users varying degrees of information and engagement. In the "insights" section of the free version, a series of topics and articles are displayed in different categories. These range from information and advice on how to balance nutrition and female health, reproductive health, self-care, cycle phases, and periods. However, not all the content within these categories is freely accessible. To gain unlimited access, users are required to upgrade to the "Premium" version. For this, payment of 9.99 Euro per month is required. While I was writing this chapter, there was also a promotion that included a 30-Day free trial, but that requires payment for the full year in advance. There the cost drops to 4.17 Euro. The app was constantly, but unsuccessfully, inviting me to unlock the premium version, which included: "a daily well-being plan, advance health assistance chats, video courses with top experts ... and more created with top medical experts" (Flo, 2020). Throughout the research, I stayed with the free version of the app.

Regarding the design and interface of Flo, I realized that contrary to the digital calendars of for example my iPhone, Outlook, or Gmail, whose interface looks gender-neutral and "serious" or "professional" in terms of the neutral colours that are used, Flo, like most of the menstrualtracking apps I initially explored, used stereotypically feminine attributes in their calendars. Pastel colour palettes, a display of images of flowers, hearts, and cartoons or photos or drawings of only young women, in their 20 s to late 30 s. Sometimes from different racial backgrounds, but mostly white. These characteristics appear to be a common trend in menstrualtracking apps (Epstein et al., 2017). Flo also assumes that the user is both a cis-gender and a heterosexual woman. This is evident for example in the wellness advice the app gives to the users and on the use of the language, being its default user a "woman", not a menstruating person, or a menstruator or ultimately, a more general term such as: a user.

\footnotetext{
${ }^{2}$ As stated above and as it will be discussed, most apps, including Flo, assume the user is both a cis-gender woman and heterosexual.
} 
I read Flo's aims with a certain degree of cynicism: "to improve the health and wellbeing of every girl and woman worldwide". Therefore,

[the app] helps women put themselves first. Flo exists to empower women by giving them a space they can access the knowledge and support they need to prioritize their health and wellbeing.

While I understand and even endorse the idea of empowerment through knowledge and prioritization of health and well-being, I consider empowerment a collective process powered by solidarity and more importantly a sense of not being alone, rather than an individual achievement. As explained by Kabeer (1994: 245-246), empowerment "entails reflection, analysis and assessment of what has hitherto been taken for granted so as to uncover the socially constructed and socially shared basis of apparently individual problems". The app "empowers women", as users collect, monitor, record, and share a range of information, that are, as Deborah Lupton (2016: 70) suggests "part of the ethical project of selfhood". I see this not as a form of empowerment, but rather as a way to become part of the quest for individual self-optimization. Digital as well as other forms of technologies should not be thought of as "[the] irreducible first cause from which social effects automatically follow" (Noble, 1979: 374).

To produce more accurate information about myself, Flo constantly requested data that was related but also unrelated to my menstrual cycleincluding sensitive personal information about my sexual life, eating and sleeping habits, overall health, and mood. I had to build a more accurate profile that, according to the app, would ultimately benefit me. But would it? The need to protect my private data accompanied me throughout this research journey and stopped me from entering any information other than the days on which I was menstruating. I only filled in data that was necessary for what the app refers to as "track cycle". Despite my stinginess in providing more information, as will be discussed later, with the little information I entered, the app offered me advice towards betterment and, for example, avoid the supposedly unwanted effects of hormones on my premenstrual body. As if there was something undesired or that should be removed from my embodied self. The app considered that premenstrual symptoms, be they feelings of discomfort, annoyance, sadness, or anger indicate a chaotic body that needs to be ordered, using the digital data to do so. 


\section{The Production of Disembodied KNOWLEDGE OF THE BODY}

I see Flo as a digital mode of knowledge production about the body. However, as contradictory as this may sound, through my experience using the app I realized that this production of knowledge of the body somehow removed my corporality and context from the equation. To illustrate this point better, I will share some posts I received through one of the apps' features called "Dive into your cycle day". This feature is designed to offer daily predictions based on the limited data I supplied to the app.

Today is your cycle day 10 . What you may experience: tender breasts, high sex drive, bloating. The hormones responsible: Two hormones dominate your body now - estrogen and luteinizing hormone (LH). They both may cause ovulation symptoms like high sex drive, bloating, and others.

Or,

Today is your cycle day 18. What you may experience: Oily skin and hair, sugar cravings strong stamina. The hormones responsible: Today the concentrations of progesterone in the blood are at their maximum, so you may feel a surge of strength and experience sugar cravings.

Or,

Today is your cycle day 22. What you may experience: Cramps, mood swings, tearfulness. The hormones responsible: The closer the menstruation gets, the less progesterone there is in the body. Estrogen levels have been low for a while. You may experience fatigue, swelling, acne, problems with the gastrointestinal tract, or mood changes.

While getting these predictions I often wondered what kind of knowledge is produced through the app and, am I benefiting from it? As a user, I noticed that the app posts served as a normative standard to measure and evaluate my physical and emotional experience throughout the menstrual cycle, inviting me to question: am I normal? Moreover, there was a constant supposition that any feelings I experience, being of joy, relaxation, happiness, annoyance, tiredness, or sadness, and changes in my physical appearance came directly from me and my hormones. While 
I think my emotions and embodied experiences are far more complex and that they are interrelated to a wider social context, I was finally able to understand the feelings of comfort and reassurance my friends spoke about when explaining their motivations to use MTAs. The app algorithms are able, for instance, to predict "emotionally volatile days" associated with the so-called Pre-Menstrual Syndrome. However, the app's predictions regarding my bodily and emotional changes did not often fit my experience. So, I found myself wondering and comparing myself again to the app's predictions: am I normal? MTAs "assume the objectivity of the data, which would result in the assumption that the bodies of similar data would have the same experience" (Çerçi, 2018: 12). Several times I wondered: "for whom are these predictions accurate?" Or in turn, "to what extent do they influence user's experiences and behaviour?" I suppose this depends on the power that a user gives to this information and the degree of trust users have with the app. It did cross my mind that it bore comparison with the horoscopes of the Ecuadorian Sunday newspaper. As with the horoscope, there was an underlying idea that the newly acquired knowledge of the app would help the reader/user to achieve fulfilment and better control of one's life. But at the same time, it could influence the reader/user's emotions and experiences in that day.

On the other hand, I enjoyed being aware or learning about the natural chemicals produced in different sites of my body and released into my bloodstream. The production of hormones is silent, painless, and odourless to my senses. Throughout my-more than a year-engagement with the app I learned and was frequently reminded about this invisible yet very powerful process of what according to the app represents the working of hormones in my body: "Higher production of estrogens today", "ovulation day", "lower production of progesterone", "rising progesterone" on this other day, "the corpus luteum has grown to it maximize size", etc. I appreciated the informative process and the awareness about what is possibly going on in there, possibly because neither the app nor I know exactly about this. But this enthusiasm was accompanied by suspicious questionings on the quantification of knowledge about me. "OK, it is fun and interesting to know about this, but, do I need to know this information?"; "What for?"; "How does it contribute to my relationship with my body and with my relationships with the people from the community to which I belong?”.

Likewise, I started to notice that these moments of acknowledgement of the chemical dimension of my cycle were quickly trivialized and 
reoriented with information on the negative side effects of, for example, low oestrogen that corresponds with the end of the menstrual cycle and possible emotions to be wary of as they might interrupt pursuits of happiness and productivity. Or, the opposite, the app described the positive side effects of high levels of oestrogen in my body and what to look forward to in the coming days. The predictions of sadness and discomfort and the incentives for happiness or success were placed on me as the individual user. These narratives fuelled by a grammar of individualism were making me, or my hormones, solely responsible for how I was feeling, replacing "notions of the social or political, or any idea of individuals as subject to pressures, constraints or even influence from the outside" (Elias \& Gill, 2018: 64).

Leaving behind the questioning on whether this information is accurate or not, I was intrigued by how predictions were framed. The language used gave me the impression of offering a precise assessment of the state of my body, as if the app knew me well or as if it had a standard to which I should compare myself. I could hardly see this as a way of reclaiming knowledge of my body. Instead, it resembled more a digitalized form of control or self-surveillance of my body. It is hard for me to condense or rather, reduce the complexity of elements that make up my understanding and experience of my body in just the flow of hormones during the different stages of my menstrual cycle. I see my body as an interconnected system of muscles, nerves, hormones, emotions, cycles, and actions and as having the capacity to memorize physical and emotional pain, joy, and pleasure. That same body is part of a web of cultural norms, power relations, beliefs, and identities. Moreover, my body's biology, its performative dimensions (Butler, 2011), and its cultural meanings are in a continuous state of change due to inner and outside factors.

\section{Final Thoughts}

In this chapter, I shared my research journey of becoming acquainted with the world of MTAs. This journey led to my encounter with Flo. I am reflexive about how my standpoint constituted my meaning-making processes while writing this chapter (Harding, 2004). The observations and reflections I presented here emerged through a process of knowledge production that resisted the notion of presenting an objective reality (Harding, 2004). I did not attempt to give a complete view of what it is to experience the use of Flo or any other MTA. This story reflects a partial 
perspective on the use of one app, which is situated in a specific moment in time, and it comes from my "specific location, embodied and particular, and never innocent" (Rose, 1997: 308). In this exploration, I had moments of tension and delight. The latter while sharing experiences with friends and family.

These moments brought me to delve into different perspectives and emotions related to the MTA and challenged some of my biases. My moments of tension were mostly connected to my concerns around privacy, which I described in "the overwhelming period". The review of the privacy policies pushed me even further regarding my distrust of how digital data is processed and handled by MTAs. Nonetheless, these moments also helped me reflect on my political motivations for doing research in this field and pointed out the need for a future exploration around ethics of care, privacy, and (datafied) bodies. Likewise, I also encountered moments of joy and intimate connection, which I explored in the stage called "Analogue intimacy". Through listening and in conversation with people with whom we shared intimate stories, I was pushed to question my own biases and expand my understanding of these digital technologies. While I understood how MTAs become tools for healthawareness and reassurance for some, my experience with Flo was different. This relates to the ways I used (not as a committed and detailed selftracker) and the ways I gave meaning to the app. In particular, that the digital data did not acquire a significant meaning in my life. I am left wondering how these predictions would have been if I had fed the app with more detailed personal data? And how at the same time, I would have related to the app, maybe more intimately?

Finally, while I wrote this chapter intending to be reflective about my journey with Flo, I am aware that there may be limits to reflexivity "both at the time of conducting it and in the years that follow" (Mauthner \& Doucet, 2003: 425). As put by Grosz (1994: 13) "the author's intentions, emotions, psyche, and interiority are not only inaccessible to readers, they are likely to be inaccessible to the author herself". What I aim to point to here is that some perceptions, feelings, and influences are hard to recognize and convey in the moment of writing. Gaining perspective through time, distance and detachment from the investigation could substantially enhance the ability to be reflexive about what I wrote in this chapter. In that sense, I see this journey with MTAs as a continuous and opened process, that will most likely change in the near future when, for instance, I revisit and reflect again on my diary notes. 


\section{REFERENCES}

Bobel, C. (2007). "I'm not an activist, though I've done a lot of it": Doing activism, being activist and the "perfect standard" in a contemporary movement. Social Movement Studies, 6, 147-159.

Butler, J. (2011). Bodies that matter: On the discursive limits of sex. Taylor \& Francis.

Çerçi, S. (2018). Embodying self-tracking: A feminist exploration of collective meaning-making of self-tracking data. M.A. thesis, Malmö University.

Elias, A. S., \& Gill, R. (2018). Beauty surveillance: The digital self-monitoring cultures of neoliberalism. European Journal of Cultural Studies, 21(1), 59-77.

Ellingson, L. L. (1998). "Then You Know How I Feel”: Empathy, identification, and reflexivity in fieldwork. Qualitative Inquiry, 4(4), 492-514.

Ellingson, L. L. (2006). Embodied knowledge: Writing researchers' bodies into qualitative health research. Qualitative health research, 16(2), 298-310.

Ellingson, L. L. (2017). Embodiment in qualitative research. Taylor \& Francis.

Epstein, D., Lee, N., Kang, J., Agapie, E. S., \& Munson, S., (2017). Examining menstrual tracking to inform the design of personal informatics tools. In Proceedings of the 2017 CHI Conference on Human Factors in Computing Systems.

Flo. (2020). Flo Health o Period tracker. My Ovulation Calendar. Retrieved from https://play.google.com/store/apps/details?id=org.iggyme dia.periodtracker. Retrieved 04 October 2020.

Gaybor, J. (2019). Empowerment, destigmatization and sustainability: The coconstruction of reusable menstrual technologies in the context of menstrual activism in Argentina. Gender, Technology and Development, 23(2), 111-129.

Gaybor, J. (2020). Everyday (online) body politics of menstruation. Feminist Media Studies, 20(1), 1-16.

Grosz, E. (1994). Volatile bodies: Towards a corporeal feminism. Indiana University Press.

Harding, S. (2004). The feminist standpoint theory reader: Intellectual and political controversies. Routledge.

Jones, S., \& Woglom, J. F. (2015). Behind the Body-Filled Scenes: Methodologies at work on the body in graphica. In P. Mia \& M. Carmen Liliana (Eds.), Methodologies of Embodiment: Inscribing Bodies in Qualitative Research (1st ed.). (pp. 116-137). Routledge. https://doi.org/10.4324/978020358 2190.

Kabeer, N. (1994). Reversed realities: Gender hierarchies in development thought. Verso.

Karlsson, A. (2019). A room of one's own?: Using period trackers to escape menstrual stigma. Nordicom Review, 40(s1), 111-123. 
Kaur, R. (2015). Facebook post 25 March. [Online] Retrieved October 22, 2020, from https://www.facebook.com/rupikaurpoetry/posts/821304554 630489

Letherby, G. (2002). Claims and disclaimers: Knowledge, reflexivity and representation in feminist research. Sociological Research Online, 6(4), 81-93.

Lupton, D. (2015). Quantified sex: A critical analysis of sexual and reproductive self-tracking using apps. Culture, Health \& Sexuality, 17(4), 440-453.

Lupton, D. (2016). Mastering your fertility. The digitised reproductive citizen in A. McCosker, S. Vivienne, \& A. Johns (Eds.), Negotiating Digital Citizenship: Control, Contest and Culture, 81-93.

Mahdawi, A. (2019, September 14). Your period-tracking app could be sharing intimate details with all of Facebook in The Guardian. Retrieved November 20, 2020, from https://www.theguardian.com/world/commentisfree/ 2019/sep/14/your-period-tracking-app-could-be-sharing-intimate-detailswith-all-of-facebook

Mauthner, N. S., \& Doucet, A. (2003). Reflexive accounts and accounts of reflexivity in qualitative data analysis. Sociology, 37(3), 413-431.

Noble, D. (1979). Social choice in machine design: The case of automatically controlled machine tools. In A. Zimbalist (Ed.), Case studies on the labour process. Monthly Review Press.

Privacy International. (2018). Investigating Apps interactions with Facebook on Android. Retrieved November 15, 2020, from https://privacyinternational. org/appdata

Privacy International. (2020). No body's business but mine: How menstruation apps are sharing your data. Retrieved November 15, 2020, from https://pri vacyinternational.org/long-read/3196/no-bodys-business-mine-how-menstr uations-apps-are-sharing-your-data

Rose, G. (1997). Situating knowledges: positionality, reflexivities and other tactics. Progress in human geography, 21(3), 305-320.

Sanders, R. (2017). Self-tracking in the digital era: Biopower, patriarchy, and the new biometric body projects. Body \& Society, 23(1), 36-63.

Setton, R., Tierney, C., \& Tsai, T. (2016). The accuracy of web sites and cellular phone applications in predicting the fertile window. Obstetrics \& Gynecology, $128(1), 58-63$.

Shaw, F. (2016). " "bitch I Said Hil": The bye felipe campaign and discursive activism in mobile dating apps." Social Media+Society, 2(4), 1-10.

Shipp, L., \& Blasco, J. (2020). How private is your period?: A systematic analysis of menstrual app privacy policies. Proceedings on Privacy Enhancing Technologies, 2020(4), 491-510.

Sörensson, E., \& Kalman, H. (2018). Care and concern in the research process: Meeting ethical and epistemological challenges through multiple engagements and dialogue with research subjects. Qualitative Research, 18(6), 706-721. 
Statista. (2020). Number of apps available in leading app stores as of 4th quarter 2020. Retrieved February 2021, from https://www.statista.com/statistics/ 276623/number-of-apps-available-in-leading-app-stores/

Thomas, G. M., \& Lupton, D. (2015). Threats and thrills: Pregnancy apps, risk and consumption. Health, Risk of Society, 17(7-8), 495-509.

Willemse, K. (2014, March) "Everything I told you was true": The biographic narrative as a method of critical feminist knowledge production. In Women's Studies International Forum (Vol. 43, pp. 38-49). Pergamon.

Open Access This chapter is distributed under the terms of the Creative Commons Attribution 4.0 International License (http://creativecommons.org/ licenses/by/4.0/), which permits use, duplication, adaptation, distribution and reproduction in any medium or format, as long as you give appropriate credit to the original author(s) and the source, a link is provided to the Creative Commons license and any changes made are indicated.

The images or other third party material in this chapter are included in the work's Creative Commons license, unless indicated otherwise in the credit line; if such material is not included in the work's Creative Commons license and the respective action is not permitted by statutory regulation, users will need to obtain permission from the license holder to duplicate, adapt or reproduce the material.

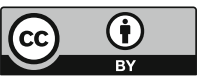

